\title{
Design of Controllers for Electrical Power Systems Using a Complex Root Locus Method
}

\author{
Arnau Dòria-Cerezo, and Marc Bodson, Fellow, IEEE
}

\begin{abstract}
A large class of three-phase electrical power systems possess symmetry conditions that make it possible to describe their behavior using single-input single-output transfer functions with complex coefficients. In such cases, an extended root locus method can be used to design control laws, even though the actual systems are multi-input multi-output. In this paper, the symmetric conditions for a large class of power systems are analyzed. Then, the root locus method is revisited for systems with complex coeffcients and used for the analysis and control design of power systems. To demonstrate the benefits of the approach, the paper includes two examples: a doubly-fed induction machine and a three-phase LCL inverter.
\end{abstract}

Index Terms-Root-locus rules, complex coefficients, power systems.

\section{INTRODUCTION}

The root locus method was developed by W.R. Evans in the 40's [1][2]. It is a fundamental tool that is taught in most, if not all, introductory courses on feedback systems. Root locus rules are commonly used by engineers when designing control systems and many references can be found in the literature for electrical and electronic applications. Examples include electronic power converters connected to the grid [3][4], STATCOMs [5], multilevel systems for renewable applications [6] or current-source inverters [7], and electrical machines such as permanent-magnet synchronous machine drives [8] or doubly-fed induction generators connected to the grid [9].

The use of a conventional root locus method implies that one considers either a single-input single-output system, or a multivariable system that is decoupled (possibly through feedforward or feedback action) so that it can be controlled as a set of single-input single-output systems. In the approach of this paper, a root locus approach is used for a broader class of systems. To achieve the result, a balanced three-phase system is first reduced to a two-phase system through a 32 transformation, and then into a single-phase system using a real to complex transformation. The last transformation is valid if the system satisfies symmetry conditions that are often applicable for electrical power systems [10]. Using this approach,

Manuscript received May 26, 2015; revised October 1, 2015 and December 10, 2015; accepted December 22, 2015.

Copyright (c) 2016 IEEE. Personal use of this material is permitted. However, permission to use this material for any other purposes must be obtained from the IEEE by sending a request to pubs-permissions@ieee.org.

A. Dòria-Cerezo is partially supported by the spanish Ministerio de Educación project DPI2013-41224-P and the catalan AGAUR project 2014 SGR 267.

A. Dòria-Cerezo is with the Department of Electrical Engineering and the Institute of Industrial and Control Engineering, Universitat Politècnica de Catalunya, 08034 Barcelona, Spain (e-mail: arnau.doria@upc.edu).

M. Bodson is with the Department of Electrical and Computer Engineering, University of Utah, Salt Lake City, UT 84112 USA (e-mail: bodson@eng.utah.edu). applications were found where the analysis of the systems could be simplified considerably. The systems included an induction motor [11], a self-excited induction generator [12], a doubly-fed induction machine with active/reactive power control [13] and microgrids [14].

Because physical systems are described by state-space models or transfer functions with real parameters, the theory of systems and control concerns almost exclusively systems with real coefficients. Few examples of dynamic systems having transfer functions with complex coefficients can be found in asymmetric bandpass and band-rejection filters [15], mobile radio communication filtering algorithms [16], whirling shafts [17], and some mechanical systems [18]. Control theory tools for systems with complex coefficients are also very limited. For example, the paper [19] provides a Hurwitz test for complex polynomials and has been applied in [12][13] (see also [20][21][22][23]). Also, extended versions of Kharitonov's criterion for polynomials with uncertain complex coefficients were studied in [17][24][25][26][27][28].

Recently, the root locus rules were derived for systems for complex coefficients [29] and general principles of symmetric systems were discussed in [30]. In this paper, we show through two examples how the complex root locus rules can be used for the design of controllers for power systems. The results are non trivial because, although the design is performed on a singleinput single-output system, the actual feedback system is a $2 \times 2$ multivariable system. As a result, the root locus exhibits characteristics not found in a conventional design, and offers possibilities that are not available in the real case.

The paper is organised as follows. In Section II the systems with symmetric properties and their complex representation are introduced. Then, in Section III, symmetric properties are used to describe power systems as systems with complex coefficients and the root locus rules for those kind systems are described in Section IV. Finally, sections V and VI include two examples (a doubly-fed induction machine and an LCL inverter, respectively), where the root locus rules are used to analyze the stability of already known controllers and to propose new algorithms that improve the performance and robustness with respect to the existing ones.

\section{COMPLEX REPRESENTATION OF SYMMETRIC SYSTEMS}

Linear systems with a special type of symmetry property were described in [10]. A symmetric system is defined as a system having a state-space representation

$$
\frac{\mathrm{d} x}{\mathrm{~d} t}=A x+B u, \quad y=C x
$$


where the state, input, and output vectors can be divided into two vectors of equal dimensions such that

$$
\begin{aligned}
& x=\left(\begin{array}{l}
x_{1}(t) \\
x_{2}(t)
\end{array}\right) \in \mathbb{R}^{2 n}, \quad u=\left(\begin{array}{l}
u_{1}(t) \\
u_{2}(t)
\end{array}\right) \in \mathbb{R}^{2(n-m)}, \\
& y=\left(\begin{array}{l}
y_{1}(t) \\
y_{2}(t)
\end{array}\right) \in \mathbb{R}^{2(n-m)}
\end{aligned}
$$

and the associated submatrices of $A, B$, and $C$ have the structure

$$
\begin{aligned}
A & =\left(\begin{array}{cc}
A_{11} & -A_{21} \\
A_{21} & A_{11}
\end{array}\right), \quad B=\left(\begin{array}{cc}
B_{11} & -B_{21} \\
B_{21} & B_{11}
\end{array}\right), \\
C & =\left(\begin{array}{cc}
C_{11} & -C_{21} \\
C_{21} & C_{11}
\end{array}\right) .
\end{aligned}
$$

An important property of a symmetric system is that it can be represented as a complex system

$$
\frac{\mathrm{d} x_{c}}{\mathrm{~d} t}=A_{c} x_{c}+B_{c} u_{c}, \quad y_{c}=C_{c} x_{c}
$$

where the complex vectors are

$$
x_{c}=x_{1}+j x_{2}, \quad u_{c}=u_{1}+j u_{2}, \quad y_{c}=y_{1}+j y_{2}
$$

and the complex matrices are

$$
A_{c}=A_{11}+j A_{21}, \quad B_{c}=B_{11}+j B_{21}, \quad C_{c}=C_{11}+j C_{21} .
$$

Note that the complex system (4) has the half of the number of states, inputs, and outputs of the original system (1), i.e., $x_{c} \in \mathbb{R}^{n}$, and $u_{c}, y_{c} \in \mathbb{R}^{n-m}$. In addition, it is proved in [12] that each root of $\operatorname{det}\left(s I-A_{c}\right)=0$ is a root of $\operatorname{det}(s I-A)=0$ and each root of $\operatorname{det}(s I-A)=0$ is represented in the roots of $\operatorname{det}\left(s I-A_{c}\right)=0$, either as itself or as its complex conjugate. Thus, knowledge of eigenvalues of the complex matrix $A_{c}$ is equivalent to knowledge of the eigenvalues of the real matrix $A$, which implies that the poles of the original system (1) can be obtained from the poles of the complex system (4), and vice-versa.

Additionally, a similar relationship appears between the transfer function matrices of the original system

$$
H(s)=C(s I-A)^{-1} B=\left(\begin{array}{cc}
H_{11}(s) & -H_{21}(s) \\
H_{21}(s) & H_{22}(s)
\end{array}\right)
$$

and the one corresponding to the complex system (4),

$$
H_{c}(s)=C_{c}\left(s I-A_{c}\right)^{-1} B_{c}=H_{11}(s)+j H_{21}(s) .
$$

See [29] for further details.

The root locus method is usually applied to closed-loop systems. Similarly to the general case for systems with real coefficients, the rules can be used for systems with complex description. To illustrate the approach, let us consider that system (1) is controlled by an static output feedback. The root locus rules presented in Section IV can be used in systems with the form (1) having 2 inputs and 2 outputs and the output feedback control law

$$
u(t)=-k\left(\begin{array}{cc}
k_{R} & -k_{I} \\
k_{I} & k_{R}
\end{array}\right) y(t)
$$

where $k, k_{R}, k_{I}$ are adjustable gains. Using a complex notation

$$
u_{c}(t)=-k k_{C} y_{c}(t)
$$

where $k_{C}=k_{R}+j k_{I}$. Then, the closed loop poles of (1) with the feedback law (9) can be obtained from the poles of (4) together with (10). Letting

$$
H_{c}(s)=\frac{N_{O L}(s)}{D_{O L}(s)},
$$

the poles can also be determined from the roots of the polynomial

$$
D_{C L}(s)=D_{O L}(s)+k k_{C} N_{O L}(s) .
$$

\section{SYMMETRIC PROPERTIES AND COMPLEX REPRESENTATION OF POWER SYSTEMS}

Many balanced three-phase electrical systems satisfy the symmetric property introduced in the previous section. This fact implies that such systems can be represented using complex coefficients and, consequently, are suited for applying the tools presented in the next sections. This section shows how any three-phase RLC circuit can be represented in the symmetric form (1) with the property (3), or as a complex system as in (4). A simple example is included, and some applications are given in Sections V and VI.

Consider a set of balanced electrical variables, $\left(f_{a}, f_{b}, f_{c}\right)=$ $F\left(\cos (\omega t), \cos \left(\omega t-\frac{2 \pi}{3}\right), \cos \left(\omega t+\frac{2 \pi}{3}\right)\right)$, and apply an $a b c$ to $\alpha \beta$ transformation $^{1}$

$$
\left(\begin{array}{l}
f_{\alpha}(t) \\
f_{\beta}(t) \\
f_{0}(t)
\end{array}\right)=\sqrt{\frac{2}{3}}\left(\begin{array}{ccc}
1 & -\frac{1}{2} & -\frac{1}{2} \\
0 & \frac{\sqrt{3}}{2} & -\frac{\sqrt{3}}{2} \\
\frac{1}{\sqrt{2}} & \frac{1}{\sqrt{2}} & \frac{1}{\sqrt{2}}
\end{array}\right)\left(\begin{array}{l}
f_{a}(t) \\
f_{b}(t) \\
f_{c}(t)
\end{array}\right) .
$$

Since the signals are balanced, the homopolar component is zero $\left(f_{0}(t)=0\right)$ and the $\alpha$ and $\beta$ components are in quadrature. Then, the $\alpha$ and $\beta$ components can be represented as a complex signal [31] as

$$
f_{\alpha \beta}=f_{\alpha}+j f_{\beta}=F_{\alpha \beta} e^{j \omega t}
$$

where $e^{j \omega t}=\cos \omega t+j \sin \omega t$ and $F_{\alpha \beta}=\sqrt{\frac{2}{3}} F$. The dqcomponents can be defined as,

$$
f_{d q}=e^{-j \theta} f_{\alpha \beta}
$$

where $\frac{\mathrm{d} \theta}{\mathrm{d} t}=\omega$. Note that the representation of the three-phase variables as a single complex variable $f_{\alpha \beta}$ or $f_{d q}$ is possible for arbitrary variables provided that the homopolar component is zero.

Three-phase RLC electrical circuits can generally be described as

$$
\left[M \otimes I_{3}\right] \frac{\mathrm{d} x}{\mathrm{~d} t}=\left[(J-D) \otimes I_{3}\right] x+\left[G \otimes I_{3}\right] u
$$

where $x^{T}=\left(x_{1}, x_{2}, \ldots, x_{p}\right) \in \mathbb{R}^{3 p}$ and $x_{i}=\left(x_{i a}, x_{i b}, x_{i c}\right)$, are the set of voltage capacitors and inductor currents, $\otimes$ denotes the Kronecker's product and $I_{3}$ is the $3 \times 3$ identity matrix. The $M$ matrix contains the capacitances and inductances values, $J$ describes the interconnection among the different three-phase components, $D$ matrix corresponds to the dissipative elements (resistance and conductances) of the circuit and $u$ contain the voltage and current sources which

\footnotetext{
${ }^{1}$ As example, we take in (13) a power-preserving transformation.
} 


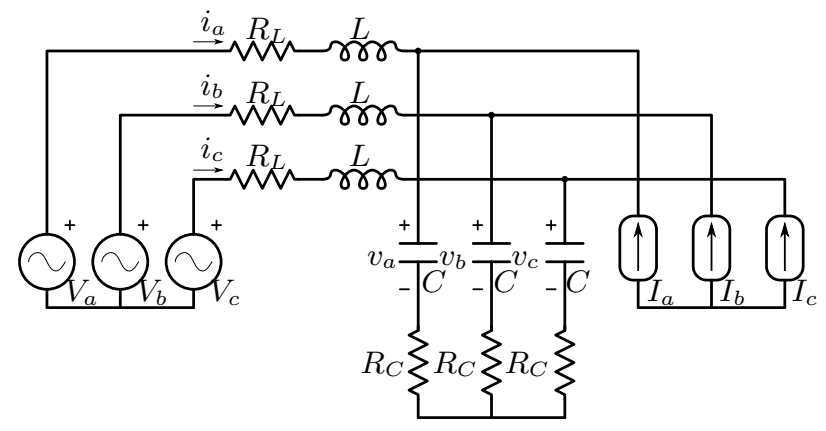

Fig. 1. Three-phase circuit example.

are connected through the $G$ matrix. For simplicity, assume that mutual inductances are neglected, so that the $M$ matrix is diagonal, $J$ is skew-symmetric $\left(J=-J^{T}\right), D$ is symmetric and positive-definite $\left(D=D^{T}>0\right)$.

Using (13), the balanced three-phase system (16) can be reduced to an $\alpha \beta$ equivalent representation

$$
M \frac{\mathrm{d} x_{\alpha \beta}}{\mathrm{d} t}=(J-D) x_{\alpha \beta}+G u_{\alpha \beta}
$$

where $x_{\alpha \beta} \in \mathbb{C}^{p}$, the matrix $M_{\alpha \beta}$ is diagonal, $J_{\alpha \beta}$ is skewsymmetric, and $D_{\alpha \beta}$ symmetric and positive-definite, and each phase is represented by a real coefficient. The dq-coordinates can be obtained defining

$$
x_{\alpha \beta}=T x_{d q}
$$

with

$$
T\left(\theta_{1}, \theta_{2}, \ldots, \theta_{p}\right)=\operatorname{diag}\left\{e^{j \theta_{1}}, e^{j \theta_{2}}, \ldots, e^{j \theta_{p}}\right\}
$$

where $\theta_{1}, \theta_{2}, \ldots, \theta_{p}$ are the instantaneous phase of each threephase signal. For simplicity, consider the case where all the signals have the same frequency, $\theta_{1}, \theta_{2}, \ldots, \theta_{p}=\theta$, and

$$
T(\theta)=e^{j \theta} I_{p}
$$

where $I_{p}$ is the $p \times p$ identity matrix. Then, using (18) and its the time derivative in (17)

$$
M\left(e^{j \theta} \frac{\mathrm{d} x_{d q}}{\mathrm{~d} t}+\frac{\partial e^{j \theta}}{\partial \theta} \omega x_{d q}\right)=(J-D) e^{j \theta} x_{d q}+G_{d q} e^{j \theta} u_{d q}
$$

and, premultiplying by $e^{-j \theta}$ the dq-system yields

$$
M \frac{\mathrm{d} x_{d q}}{\mathrm{~d} t}=(J-D-j \omega M) x_{d q}+G_{d q} u_{d q}
$$

where the fact that $e^{-j \theta} \frac{\partial e^{j \theta}}{\partial \theta}=j$ has been used.

Note that when the transformation (18) is applied in (16), complex parameters appear in the system.

The dq-representation in (22) is still valid for non-linear systems and, in that case, matrices would not be longer constant. However, the transfer function representation proposed in II will be only valid for linear ones. In addition, using the decoupling of symmetric components for unbalanced systems, the presented methodology can be applied to unbalanced power systems, through the use of two complex models corresponding to the positive and negative sequences [10][31]. See example in Subsection VI-D.
Example: Consider the three-phase circuit shown in Fig. 1. Its model can be written in the form (16) with

$$
\begin{aligned}
& M=\left(\begin{array}{ll}
L & 0 \\
0 & C
\end{array}\right), \quad J=\left(\begin{array}{cc}
0 & -1 \\
1 & 0
\end{array}\right) \\
& D=\left(\begin{array}{cc}
R_{L}+R_{C} & 0 \\
0 & 0
\end{array}\right), \quad G=\left(\begin{array}{cc}
1 & -R_{C} \\
0 & 1
\end{array}\right)
\end{aligned}
$$

where $x=\left(i_{L}, v_{C}\right)^{T}, i_{L}$ and $v_{C}$ are the three-phase inductor currents and capacitor voltages, and $u=(v, i)^{T}$ are the three-phase voltage and current sources. Then, the space-state description with complex coefficients is given by

$$
\frac{\mathrm{d} x_{d q}}{\mathrm{~d} t}=\left(\begin{array}{cc}
-\frac{R_{L}+R_{C}}{L}-j \omega & -\frac{1}{L} \\
\frac{1}{C} & -j \omega
\end{array}\right) x_{d q}+\left(\begin{array}{cc}
\frac{1}{L} & -\frac{R_{C}}{L} \\
0 & \frac{1}{C}
\end{array}\right) u_{d q}
$$

which follows the form of (4). The system can be described by

$$
A_{O L}(s)\left(\begin{array}{c}
i_{L} \\
v_{C}
\end{array}\right)=\left(\begin{array}{c}
v-R_{C} i \\
i
\end{array}\right)
$$

where

$$
A_{O L}(s)=\left(\begin{array}{cc}
R_{L}+R_{c}+(s+j \omega) L & -1 \\
1 & (s+j \omega) C
\end{array}\right) .
$$

\section{ROOT LOCUS RULES FOR SYSTEMS WITH COMPLEX COEFFICIENTS}

The root locus rules characterize the movement of the closed-loop poles of a system as a function of a varying parameter, $k$. In the case of a system with complex coefficients, the polynomial under consideration is of the from

$$
D_{C L}(s)=D_{O L}(s)+k k_{C} N_{O L}(s)
$$

where $k>0$ is the variable gain and $k_{C}=k_{R}+j k_{I}$. In general, the polynomials $D_{O L}(s)$ and $N_{O L}(s)$ can be represented as

$$
\begin{aligned}
D_{O L}(s) & =\left(s-p_{1}\right)\left(s-p_{2}\right) \ldots\left(s-p_{n}\right)= \\
& =s^{n}+a_{1} s^{n-1}+\ldots+a_{n-1} s+a_{n} \\
N_{O L}(s) & =\left(s-z_{1}\right)\left(s-z_{2}\right) \ldots\left(s-z_{m}\right)= \\
& =s^{m}+b_{1} s^{m-1}+\ldots+b_{m-1} s+b_{m} .
\end{aligned}
$$

Note that, because the coefficients of the polynomials are complex, the poles $p_{i}$ and zeros $z_{i}$ do not have to appear as complex pairs.

By definition, the root locus is the locus of the roots of the characteristic equation $D_{C L}(s)=0$

$$
D_{O L}(s)+k k_{C} N_{O L}(s)=0 .
$$

as $k$ varies from 0 to infinity. For convenience, we will refer to the real root locus as the locus of a system with polynomials $N_{O L}(s)$ and $D_{O L}(s)$ having real coefficients and $k_{C}=1$, and to the complex root locus when either of the polynomials $N_{O L}(s), D_{O L}(s)$ has complex coefficients, and/or $k_{C}$ is an arbitrary complex number.

The root locus rules for system with complex coefficients were introduced in [29] and are summarized here:

1) Number of branches: The number of branches of the root locus is equal to the degree of the characteristic polynomial, $n$. 
2) Starting points of the root locus: The root locus of $D_{C L}(s)$ starts at the open-loop poles, $D_{O L}(s)=0$.

3) End points of the root locus: $m$ of the branches of the root locus converge to the roots of $N_{O L}(s)$, while the other $n-m$ roots converge to infinity along asymptotes whose angles with respect to the real axis are defined by the $n-m$ complex roots of

$$
s_{i}=\sqrt[n-m]{-k_{C}}, \quad i=1, \ldots, n-m .
$$

The center of the asymptotes is located at

$$
c=\frac{1}{n-m}\left(\sum_{i=1}^{n} p_{i}-\sum_{i=1}^{m} z_{i}\right) .
$$

4) Break-away points: A break-away point $s_{0}$ must be a root of (33) that also fulfills

$$
\frac{\left.\frac{\mathrm{d} D_{O L}(s)}{\mathrm{d} s}\right|_{s=s_{0}}}{\left.\frac{\mathrm{d} N_{O L}(s)}{\mathrm{d} s}\right|_{s=s_{0}}}=\frac{\left.D_{O L}(s)\right|_{s=s_{0}}}{\left.N_{O L}(s)\right|_{s=s_{0}}} .
$$

5) Angle of departure from complex poles: The angle of departure from a complex pole, $p_{j}$, of $D_{O L}(s)$ is given by

$$
\theta_{j}^{d}=\pi+\angle k_{C}+\sum_{i=1}^{m} \angle\left(p_{j}-z_{i}\right)-\sum_{i=1, i \neq j}^{n} \angle\left(p_{j}-p_{i}\right) .
$$

6) Angle of arrival at complex zeros: The angle of arrival at a complex zero, $z_{j}$, of $N_{O L}(s)$ is given by

$$
\theta_{j}^{a}=\pi-\angle k_{C}-\sum_{i=1, i \neq j}^{m} \angle\left(z_{j}-z_{i}\right)+\sum_{i=1}^{n} \angle\left(z_{j}-p_{i}\right) .
$$

7) Imaginary axis crossing: The intersection of the root locus with the imaginary axis can be found by separating the equation

$$
D_{O L}(j \omega)+k k_{C} N_{O L}(j \omega)=0
$$

into real and imaginary parts and finding values of $k$ for which real solutions exist for $\omega$. Alternatively, the values for $k$ can be obtained from the complex Hurwitz test given in [19].

Overall, the complex root locus exhibits striking similarities with the real root locus. However, peculiar differences are also observed, namely:

- the root-locus, including the asymptotes, is not necessarily symmetric with respect to the real axis;

- the center of the asymptotes does not have to be real;

- break-away points are less common than in the real root locus, because no portion of the real axis typically belongs to the root locus;

These differences in properties are possible because the complex root locus is, in general, associated with a multi-input multi-output feedback system.

\section{EXAMPLE: A DOUBLY-FED INDUCTION MACHINE}

In this section, the complex root locus rules are applied for the analysis of a feedback-linearizing current controller for a DFIM and then used to modify the control algorithm improving the performance and robustness of the resulting closed loop system. The dq transformation for a DFIM is applied for the stator and rotor variables independently, i.e., the angle in (15) is different for the stator, $\theta_{s}$, and rotor transformation, $\theta_{r}$. The so-called synchronous reference frame eliminates the dependency of the model on the rotor position by taking $\theta_{s}, \theta_{r}$ such that $\frac{\mathrm{d} \theta_{s}}{\mathrm{~d} t}=\omega_{s}$ and $\frac{\mathrm{d} \theta_{r}}{\mathrm{~d} t}=\omega_{s}-\omega_{m}$, where $\omega_{s}$ is the stator voltage frequency and $\omega_{m}$ is the mechanical speed. See further details in [32]. Then, similarly to (22), the dq-current dynamics of the DFIM can be described as

$M \frac{\mathrm{d} x}{\mathrm{~d} t}=\left(\begin{array}{cc}-R_{s}-j \omega_{s} L_{s} & -j \omega_{s} L_{s r} \\ -j\left(\omega_{s}-\omega_{m}\right) L_{s r} & -R_{r}-j\left(\omega_{s}-\omega_{m}\right) L_{r}\end{array}\right) x+G u$

where $x=\left(i_{s}, i_{r}\right)^{T}=\left(i_{s d}+j i_{s q}, i_{r d}+j i_{r q}\right)^{T}$ are the dq-currents in the stator and rotor (subindices $s$ and $r$, respectively), $u=\left(v_{s}, v_{r}\right)^{T}=\left(v_{s d}+j v_{s q}, v_{r d}+j v_{r q}\right)^{T}$ are the stator and rotor voltages, $R_{s}, R_{r}$ are is the stator and rotor resistances, $L_{s}, L_{r}$ and $L_{s r}$ are the stator and rotor self-inductances and mutual inductance, respectively, and the matrices $M$ and $G$ are

$$
M=\left(\begin{array}{cc}
L_{s} & L_{s r} \\
L_{s r} & L_{r}
\end{array}\right), \quad G=\left(\begin{array}{ll}
1 & 0 \\
0 & 1
\end{array}\right) .
$$

All the parameters are positive.

The stator voltage, $v_{s}$ is assumed to be set by the power grid, and the rotor voltage $v_{r}$ is used as a control input to regulate the active and reactive powers produced. Assuming a fixed stator voltage, the regulation of the two powers is equivalent to the tracking of a complex stator current reference $i_{s}^{\text {ref }}$.

\section{A. Feedback linearizing control law}

In this section we use the feedback linearizing control law for the DFIM proposed in [13]. The main benefit of using an algorithm that linearizes the closed loop system is that the resulting one is linear and the root locus method presented in Section IV can be used. This control law in [13] is, in a complex notation,

$$
\begin{aligned}
& v_{r}=j\left(\omega_{s}-\omega_{r}\right) L_{s r} i_{s}+\left(R_{r}+j\left(\omega_{s}-\omega_{r}\right) L_{r}\right) i_{r}+\hat{v}_{r} \\
& \hat{v}_{r}=j k_{P}\left(i_{s}^{\mathrm{ref}}-i_{s}\right)+j k_{P} \frac{1}{T_{i}} \int_{t_{0}}^{t}\left(i_{s}^{\mathrm{ref}}-i_{s}\right) \mathrm{d} \tau
\end{aligned}
$$

where $k_{P}$ and $T_{i}$ are control gains. The closed-loop system (40) with (42)-(43) can be described by

$$
A_{C L}(s)\left(\begin{array}{c}
i_{s} \\
i_{r} \\
\hat{v}_{r}
\end{array}\right)=\left(\begin{array}{c}
v_{s} \\
0 \\
j k_{P}\left(s+\frac{1}{T_{i}}\right) i_{s}^{\mathrm{ref}}
\end{array}\right)
$$

where the matrix $A_{C L}(s)$ is given by

$$
A_{C L}(s)=\left(\begin{array}{ccc}
L_{s} s+R_{s}+j \omega_{s} L_{s} & L_{s r} s+j \omega_{s} L_{s r} & 0 \\
L_{s r} s & L_{r} s & -1 \\
j k_{P}\left(s+\frac{1}{T_{i}}\right) & 0 & s
\end{array}\right) .
$$

Due to the feedback linearization terms, $A_{C L}(s)$ does not depend on the mechanical speed, $\omega_{m}$. The complex polynomial $D_{C L}(s)=\operatorname{det} A_{C L}(s)$ is

$$
D_{C L}(s)=a_{0} s^{3}+\left(a_{1}+j b_{1}\right) s^{2}+\left(a_{2}+j b_{2}\right) s+a_{3}+j b_{3}
$$


where

$$
\begin{array}{llrl}
a_{3} & =\frac{k_{P}}{T_{i}} \omega_{s} L_{s r} & b_{3} & =0 \\
a_{2} & =k_{P} \omega_{s} L_{s r} & b_{2} & =-\frac{k_{P}}{T_{i}} L_{s r} \\
a_{1} & =L_{r} R_{s} & b_{1} & =\omega_{s} \mu-k_{P} L_{s r} \\
a_{0} & =\mu & &
\end{array}
$$

and $\mu=L_{s} L_{r}-L_{s r}^{2}>0$. The complex polynomial can be written as (28) with

$$
\begin{aligned}
D_{O L}(s) & =\mu s^{3}+\left(L_{r} R_{s}+j \omega_{s} \mu\right) s^{2} \\
N_{O L}(s) & =s^{2}+\left(\frac{1}{T_{i}}+j \omega_{s}\right) s+j \frac{1}{T_{i}} \omega_{s} \\
k & =k_{P} \\
k_{C} & =-j L_{s r},
\end{aligned}
$$

where $n=3$ and $m=2$. Following the root locus rules from Section IV, we get:

1) Number of branches: From $D_{O L}(s)$ in (48), the number of branches is $n=3$.

2) Starting points of the root locus: The starting points are the roots of $D_{O L}(s)$ in (48),

$$
p_{1}=p_{2}=0, \quad p_{3}=-\frac{L_{r} R_{s}}{\mu}-j \omega_{s} .
$$

3) End points of the root locus: As $k \rightarrow \infty$, two roots converge to the roots of $N_{O L}(s)$ in (48), that are

$$
z_{1}=-\frac{1}{T_{i}}, \quad z_{2}=-j \omega_{s},
$$

and the other root converges to $\infty$ along an asymptote with an angle

$$
\theta^{\infty}=\frac{\pi}{2},
$$

and a centroid

$$
c=-\frac{L_{r} R_{s}}{\mu}+\frac{1}{T_{i}} .
$$

4) Break-away points: From (36), the following complex equation is obtained

$$
\begin{aligned}
0= & \mu T_{i} s^{3}+2 \mu\left(1+j \omega_{s} T_{i}\right) s^{2} \\
& +\left(L_{r} R_{s}-\omega_{s}^{2} \mu T_{i}+j \omega_{s}\left(T_{i} L_{r} R_{s}+4 \mu\right)\right) s \\
& -2 \omega_{s}^{2} \mu+2 j L_{r} R_{s} \omega_{s} .
\end{aligned}
$$

Consequently, break-away points exist for those values of $R_{s}, L_{r}, \mu, \omega_{s}$ and $T_{i}$ that satisfy (53) together with (46).

5) Angles of departure from the complex poles: The angles of departure from $p_{1}, p_{2}, p_{3}$ are

$$
\begin{aligned}
\theta_{1}^{d} & =\frac{\pi}{2}+\frac{1}{2} \arctan \left(\frac{\omega_{s} \mu}{L_{r} R_{s}}\right) \\
\theta_{2}^{d} & =\frac{1}{2} \arctan \left(\frac{\omega_{s} \mu}{L_{r} R_{s}}\right) \\
\theta_{3}^{d} & =\frac{\pi}{2}+\arctan \left(\frac{\omega_{s} \mu T_{i}}{L_{r} R_{s} T_{i}-\mu}\right)-2 \arctan \left(\frac{\omega_{s} \mu}{L_{r} R_{s}}\right)
\end{aligned}
$$

6) Angles of arrival at the complex zeros: The angles of arrival at $z_{1}$ and $z_{2}$ are

$$
\begin{aligned}
\theta_{1}^{a} & =-\frac{\pi}{2}+\arctan \left(\omega_{s} T_{i}\right)+\arctan \left(\frac{\omega_{s} \mu T_{i}}{L_{r} R_{s} T_{i}-\mu}\right) \\
\theta_{2}^{a} & =\frac{\pi}{2}+\arctan \left(\omega_{s} T_{i}\right) .
\end{aligned}
$$

7) Imaginary axis crossing: From (39), the following two conditions are obtained

$$
\begin{array}{r}
\omega^{2} L_{r} R_{s}-\frac{k_{P} L_{s r}}{T_{i}}\left(\omega_{s}+\omega\right)=0 \\
\mu \omega^{2}+\omega\left(\omega_{s} \mu-k_{P} L_{s r}\right)-k_{P} L_{s r} \omega_{s}=0 .
\end{array}
$$

For $T_{i}>\frac{\mu}{L_{r} R_{\mathrm{s}}}$, the conditions imply that the root locus crosses the imaginary axis for

$$
k_{P}=\frac{\omega_{s} \mu^{2}}{\left(L_{r} R_{s} T_{i}-\mu\right) L_{s r}}, \quad \omega=\frac{\omega_{s} \mu}{L_{r} R_{s} T_{i}-\mu} .
$$

The value of $k_{P}$ for crossing the imaginary axis corresponds to the stability condition given in [13].

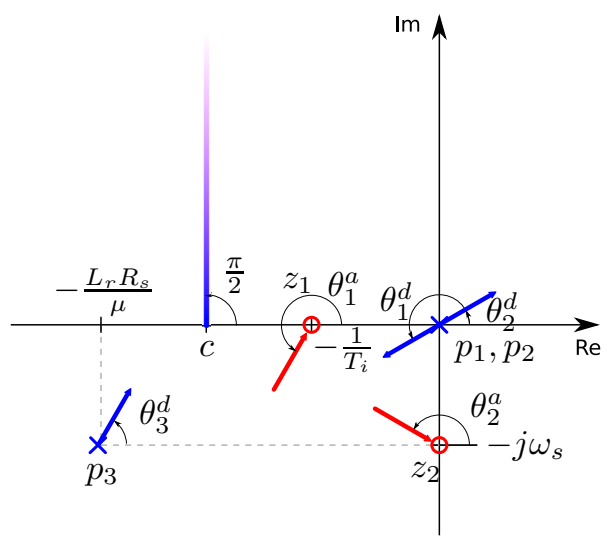

Fig. 2. Root-locus simplified scheme.

The results of the root locus rules are summarized in Fig. 2. The rules are applied to the specific DFIM parameter values given in [13]: $R_{s}=4.92, R_{r}=4.42, L_{s}=0.725, L_{r}=$ $0.715, L_{s r}=0.71$ and $\omega_{s}=314$. For those values and using (46) and (53), two break-away points appear for specific values of the parameter $T_{i}$. One occurs for a negative value of $k_{P}$ and does not belong to the (positive) root locus, and the other appears at $s=-148.5-j 177.58$ for $T_{i}=T_{i}^{\mathrm{BK}}=0.0049$. The break-away point corresponds to $k_{P}=4.82$. From the conditions obtained in Rule 7, the root locus crosses the imaginary axis for values $T_{i}=T_{i}^{\mathrm{IAC}}=0.0041$. Fig. 3 shows the root locus for several $T_{i}$ 's such that scenarios with different break-away points and imaginary axis crossings occur. In Fig. 4, a zoom of the root locus allows to clearly identify the break-away point for $T_{i}=T_{i}^{\mathrm{BK}}$ at the expected location in the complex plane.

Conclusions from the root locus are as follows:

- one of the poles at $s=0$ moves to infinity along an asymptote parallel to the $j \omega$ axis. The asymptote is in the open left-half plane for $T_{i}>0.0041$.

- for $T_{i}<0.0049$, the pole at $s=-\frac{L_{r} R_{s}}{\mu}-j \omega_{s}$ moves to the zero at $s=-\frac{1}{T_{i}}$ and one of the poles at $s=0$ moves to the zero at $s=-j \omega_{s}$. 


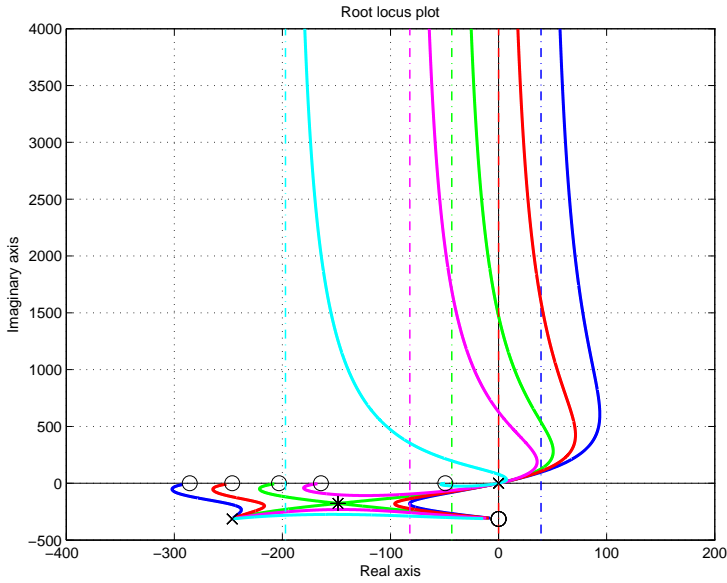

Fig. 3. Root locus of the DFIM example. The $T_{i}$ parameter takes the value: $T_{i}=0.7 T_{i}^{\mathrm{BK}}$ (blue line), $T_{i}=T_{i}^{\mathrm{IAC}}$ (green line), $T_{i}=T_{i}^{\mathrm{BK}}$ (red line), $T_{i}=1.5 T_{i}^{\mathrm{IAC}}$ (magenta line) and $T_{i}=5 T_{i}^{\mathrm{IAC}}$ (cyan line).

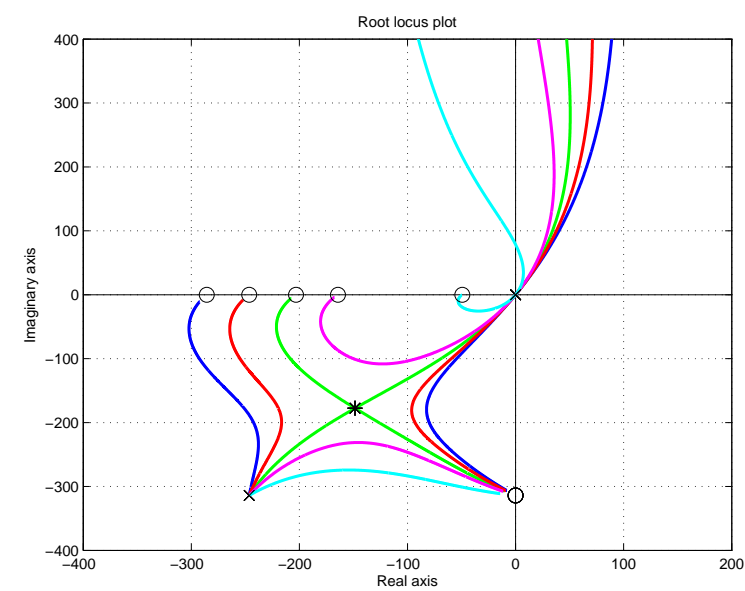

Fig. 4. Zoom of the root locus of the DFIM example. The $T_{i}$ parameter takes the value: $T_{i}=0.7 T_{i}^{\mathrm{BK}}$ (blue line), $T_{i}=T_{i}^{\mathrm{IAC}}$ (green line), $T_{i}=T_{i}^{\mathrm{BK}}$ (red line), $T_{i}=1.5 T_{i}^{\mathrm{IAC}}$ (magenta line) and $T_{i}=5 T_{i}^{\mathrm{IAC}}$ (cyan line).

- for $T_{i}>0.0049$, the pole at $s=-\frac{L_{r} R_{s}}{\mu}-j \omega_{s}$ moves to the zero at $s=-j \omega_{s}$ and one of the poles at $s=0$ moves to the zero at $s=-\frac{1}{T_{i}}$.

- for $T_{i}=0.0049$, the poles at $s=-\frac{L_{r} R_{s}}{\mu}-j \omega_{s}$ and $s=0$ merge at the break-away point, then move to the zeros at $s=-\frac{1}{T_{i}}$ and $s=-j \omega_{s}$.

- it is possible to create a stable closed-loop with the controller and degrees of freedom available, but the responses are expected to be oscillatory, and the speed of response is limited.

\section{B. Control design in the complex domain}

The analysis of the previous control design exhibits two undesirable features: the system is only conditionally stable (it becomes unstable if the gain is reduced), and two poles are poorly damped, independently to the selected gains. The use of the complex root locus can help to improve the design [30].
The poorly damped poles are associated with the two poles at $s=0$, one of which comes from the integrator in the control law and the other from the cancellation of the rotor resistance. Setting

$$
v_{r}=j\left(\omega_{s}-\omega_{r}\right) L_{s r} i_{s}+\left(k_{R} R_{r}+j\left(\omega_{s}-\omega_{r}\right) L_{r}\right) i_{r}+\hat{v}_{r}
$$

where $\hat{v}_{r}$ remains as in (43) and $k_{R}$ is a new control gain that allows one to move one pole at the origin to the left-half plane. The result is

$$
\begin{aligned}
D_{O L}(s)= & \mu s^{3}+\left(R_{s} L_{r}+\left(1-k_{R}\right) R_{r} L_{s}+j \omega_{s} \mu\right) s^{2} \\
& +\left(R_{s}+j \omega_{s} L_{s}\right)\left(1-k_{R}\right) R_{r} s
\end{aligned}
$$

and $N_{O L}(s)$ remains the same. An extreme case is obtained by setting $k_{R}=0$ so that the denominator becomes

$$
\begin{aligned}
D_{O L}(s)= & \mu s^{3}+\left(R_{s} L_{r}+R_{r} L_{s}+j \omega_{s} \mu\right) s^{2} \\
& +\left(R_{s}+j \omega_{s} L_{s}\right) R_{r} s .
\end{aligned}
$$

Now, the open-loop system only has one pole at $s=0$, while the other two poles are stable. An additional degree of freedom is obtained by exploiting the complex nature of (43) using a complex gain $k_{G}$ in

$$
\hat{v}_{r}=k_{G}\left(k_{P}\left(i_{s}^{\mathrm{ref}}-i_{s}\right)+k_{P} \frac{1}{T_{i}} \int_{t_{0}}^{t}\left(i_{s}^{\mathrm{ref}}-i_{s}\right) \mathrm{d} \tau\right) .
$$

Note that the original controller (43) is recovered with $k_{G}=$ $-j$. A different choice is to set $k_{G}=1$ which results in the asymptote becoming parallel to the real axis (see red rootlocus in Fig. 5). A real gain equal to 1 corresponds to passing the signals straight through, while a purely imaginary one introduces a crossing of the two channels.

An improved placement of the poles can be found combining (58) and (61), where one pole at $s=0$ is removed, and the angle associated with $k_{G}$ is adjusted between $\frac{\pi}{2}$ and $\pi$. Fig. 5 compares the root locus of the original control law (42)-(43), in blue, with the modified one in magenta (58)(61). Note that the controller with $k_{R}=0, k_{G}=-j$ (green root-locus) sends one pole from $s=0$ to the left side of the plane $(-64-j 147)$. In red, the root locus is displayed with the choice of $k_{G}=1$ but still cancelling $R_{r}\left(k_{R}=1\right)$. The choices result in the asymptote at $\pi$, but the range of values for stability is reduced when one of the poles starts at $s=0$. Finally, a combination of $k_{R}$ and $k_{G}$ offers a new scenario with three different poles. See in magenta the root locus for $k_{R}=0.8$ and $k_{G}=1-j 1.5$. The last set of values results in a placement of the poles at $76+j 61,-121-j 68$ and $-183-j 174$ for $k_{P}=1.8$ (marked with *'s in Fig. 5). Note that the design using the root locus properties resolved the problems found in the original design by yielding a system that is stable for all gains and pole locations associated with desirable transient responses.

Summing up, the use of the complex root locus method improved the performance (response time) and the robustness (stable for all $k$ gains and all the poles far from the positive real plane) of the system. 


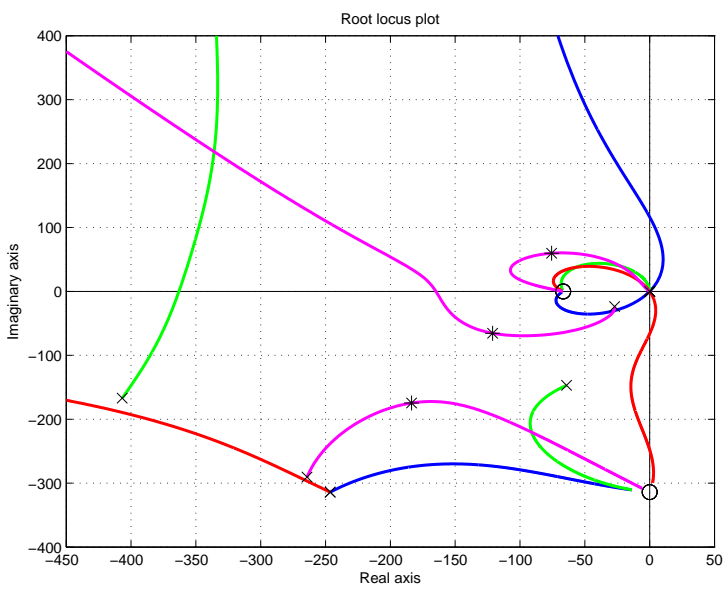

Fig. 5. Root locus comparing the proposed controllers for the DFIM. The $T_{i}$ parameter has been set at $T_{i}=0.015$. The four controller are based on (58)-(61) with: $k_{R}=1$ and $k_{G}=-j$ (in blue), $k_{R}=0$ and $k_{G}=-j$ (in green), $k_{R}=1$ and $k_{G}=1$ (in red), and $k_{R}=0.8$ and $k_{G}=1-j 1.5$ (in magenta). Marks at $*$, represent the poles for $k_{P}=1.8, k_{R}=0.8$ and $k_{G}=1-j 1.5$.

\section{EXAMPLE: A THREE-PHASE GRID INVERTER WITH AN LCL FILTER}

Similarly to the previous example, the root locus method for systems with complex coefficients has been used to compare two existing controllers for an LCL inverter and to propose an alternative control algorithm which is more robust in the presence of variations of the grid impedance.

The equations describing the dynamics of a three-phase inverter with an LCL filter in dq-coordinates are given in [33] and can be put in the form (22) with

$$
\begin{aligned}
& M=\left(\begin{array}{ccc}
L_{1} & 0 & 0 \\
0 & L_{2} & 0 \\
0 & 0 & C
\end{array}\right), \quad J=\left(\begin{array}{ccc}
0 & 0 & -1 \\
0 & 0 & 1 \\
1 & -1 & 0
\end{array}\right), \\
& D=\left(\begin{array}{ccc}
R_{1} & 0 & 0 \\
0 & R_{2} & 0 \\
0 & 0 & 0
\end{array}\right), \quad G=\left(\begin{array}{cc}
V_{d c} & 0 \\
0 & 1 \\
0 & 0
\end{array}\right) \text {, }
\end{aligned}
$$

where the state vector $x=\left(i_{1}, i_{2}, v_{c}\right)^{T}=\left(i_{1 d}+j i_{1 q}, i_{2 d}+\right.$ $\left.j i_{2 q}, v_{c d}+j v_{c q}\right)^{T}$ composed of the inverter-side currents, $i_{1}$, the grid-side currents, $i_{2}$, and the capacitor voltages, $v_{c}$, while the input vector $u=\left(u_{c}, v_{g}\right)^{T}=\left(u_{c d}+j u_{c q}, v_{g d}+j v_{g q}\right)^{T}$ is composed of the duty cycles, $u_{c}$ (which act as a control input) and the grid voltages, $v_{g}$. The parameters $R_{1}, R_{2}$ represent the losses at the inductors $L_{1}, L_{2}, C$ is the capacitance of the filter and $V_{d c}$ is the dc input voltage.

The open loop dynamics can be written as

$$
\left(\begin{array}{ccc}
N_{1}(s) & 0 & 1 \\
0 & N_{2}(s) & -1 \\
-1 & 1 & N_{c}(s)
\end{array}\right)\left(\begin{array}{c}
i_{1} \\
i_{2} \\
v_{c}
\end{array}\right)=\left(\begin{array}{c}
V_{d c} d \\
v_{g} \\
0
\end{array}\right)
$$

where

$$
\begin{aligned}
& N_{1}(s)=(s+j \omega) L_{1}+R_{1} \\
& N_{2}(s)=(s+j \omega) L_{2}+R_{2} \\
& N_{c}(s)=(s+j \omega) C .
\end{aligned}
$$

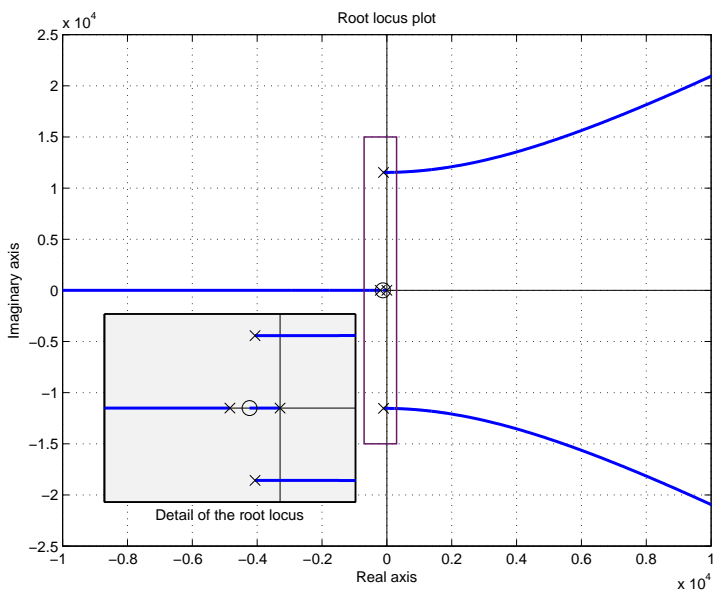

Fig. 6. Root locus of the conventional controller.

Treating $v_{g}$ as a disturbance $\left(v_{g}=0\right)$,

$$
\begin{aligned}
& v_{c}=N_{2}(s) i_{2} \\
& i_{1}=\left(N_{2}(s) N_{c}(s)+1\right) i_{2}
\end{aligned}
$$

and the (complex) transfer function of the system from the input $u_{c}$ to the output $i_{2}$ is

$$
i_{2}=\frac{V_{d c}}{D_{O L}(s)} u_{c}
$$

where

$$
D_{O L}(s)=N_{1}(s)+N_{2}(s)+N_{1}(s) N_{2}(s) N_{c}(s),
$$

or, splitting into the real and imaginary parts

$$
D_{O L}(s)=N_{r}(s)+j N_{i}(s) .
$$

\section{A. Conventional current controller}

A classical approach for controlling the LCL three-phase inverter [33] is

$$
u_{c}=j \frac{N_{i}(s)}{V_{d c}} i_{2}+\left(k_{P}+\frac{k_{I}}{s}\right)\left(i_{2}^{\mathrm{ref}}-i_{2}\right)
$$

where $k_{P}, k_{I}$ are the control design parameters and $i_{2}^{\text {ref }}$ is the grid-side dq-current references. Inserting (73) in (70), we get

$$
i_{2}=\frac{V_{d c}\left(k_{P} s+k_{I}\right)}{D_{C L}(s)} i_{2}^{\mathrm{ref}}
$$

where

$$
D_{C L}(s)=s N_{r}(s)+V_{d c}\left(k_{P} s+k_{I}\right) .
$$

Note that, because the transfer function is real, the closed-loop system is decoupled. Since the number of branches of the root locus is 4 (Rule 1), conclusions from the root locus analysis are limited. However, using Rule 3, one finds that one of the roots converges to $-\frac{k_{I}}{k_{P}}$, the other real root goes to infinity along the negative real axis, and two complex conjugate poles cross into the left half plane before reaching asymptotes at $\pm 60^{\circ}$. Fig. 6 shows the root locus. To maintain the stability of the system, the gain must be sufficiently small . 


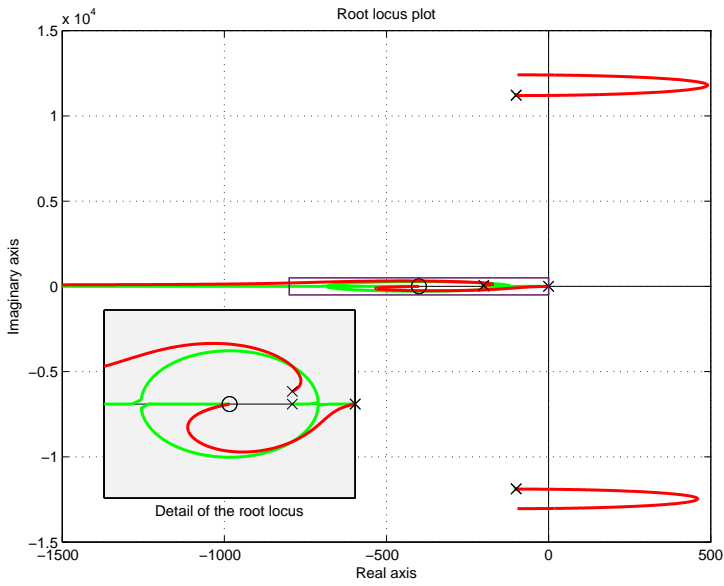

Fig. 7. Root locus comparing the WACC (in green) and the WACC with wrong parameters (in red).

\section{B. Weighted average current control}

The so-called weighted average current control (WACC) was proposed in [33]. The WACC scheme allows one to reduce the LCL filter from a third-order to a first-order system under certain assumptions. The WACC control law is

$$
u_{c}=j \omega \frac{L_{1}+L_{2}}{V_{d c}} i_{w}+\left(k_{P}+\frac{k_{I}}{s}\right)\left(i_{2}^{\mathrm{ref}}-i_{w}\right)
$$

where the weighted (complex) average current is defined by

$$
i_{w}=\frac{L_{1}}{L_{1}+L_{2}} i_{1}+\frac{L_{2}}{L_{1}+L_{2}} i_{2} .
$$

Assuming

$$
\frac{R_{1}}{L_{1}}=\frac{R_{2}}{L_{2}}=\alpha
$$

the closed-loop polynomial becomes

$$
\begin{aligned}
D_{C L}(s)= & \left(s(s+\alpha)+\frac{V_{d c}}{L_{1}+L_{2}}\left(s k_{P}+k_{I}\right)\right) \times \\
& \left(L_{1}+L_{2}+L_{1} N_{2}(s) N_{c}(s)\right) .
\end{aligned}
$$

In other words, two poles are fixed at the roots of

$$
L_{1}+L_{2}+L_{1} N_{2}(s) N_{c}(s)=0,
$$

while two other poles are determined by

$$
s(s+\alpha)+\frac{V_{d c}}{L_{1}+L_{2}}\left(s k_{P}+k_{I}\right)=0 .
$$

Due to the two poles fixed by (80), the root locus analysis reduces to the study of equation (81) which has real coefficients. Starting points are located at $0,-\alpha$ and one of these poles goes to the zero at $-\frac{k_{I}}{k_{P}}$, while the other pole goes to infinity along the negative real axis. There is no limit on the gains. The root locus shown in Fig. 7 (in green) for the WACC algorithm includes two complex poles, but they remain in the same position regardless of the control gain values due to colocation with zeros.

Assumption (78) is a significant issue with the WACC algorithm. More importantly, the benefits of the WACC are severely reduced for some types of parameter error, especially errors in the inductances $L_{1}$ and $L_{2}$ required for the calculation of $i_{w}$. Indeed, assume that $L_{2}$ varies from its nominal value, but we keep in the controller $((76))$ the estimated (and wrong) value $\hat{L}_{2}$. Then

$$
u_{c}=j \omega \frac{\hat{L}_{1}+\hat{L}_{2}}{V_{d c}} i_{w}+\left(k_{P}+\frac{k_{I}}{s}\right)\left(i_{2}^{\mathrm{ref}}-i_{w}\right)
$$

where, $i_{w}$, using (69), yields

$$
i_{w}=\frac{1}{L+\hat{L}_{2}}\left(L\left(N_{2}(s) N_{c}(s)+1\right)+\hat{L}_{2}\right) i_{2} .
$$

Fig. 7 shows how the root locus of the WACC scheme (in red) changes with a variation $L_{2}$. Unfortunately, the poles first move towards the right-half plane in this case, yielding a low gain limit guaranteeing stability. Note that the two complex poles are close to being, but are not exact complex conjugates of each other, which explains the double peaking on the frequency domain observed in [33]. Also, one of the real poles now has a small imaginary component, and both real poles move slightly off the real axis.

\section{Control design in the complex domain}

An alternate control law based on the two previous concepts is

$$
u_{c}=j \frac{N_{i}(s)}{V_{d c}} i_{2}-k_{G} i_{1}+\left(k_{P}+\frac{k_{I}}{s}\right)\left(i_{2}^{\mathrm{ref}}-i_{2}\right)
$$

which results in the transfer function (74) with

$D_{C L}(s)=s M(s)+s V_{d c} k_{G}\left(N_{2}(s) N_{c}(s)+1\right)+V_{d c}\left(k_{P} s+k_{I}\right)$.

Fig. 8 shows (in magenta) the root locus of the alternate control law, varying the gains $k_{P}$ and $k_{I}$ simultaneously. One of the real poles goes to the zero at $-\frac{k_{I}}{k_{P}}$, the other real pole goes to infinity along the negative real axis, and two complex poles cross to the left-half plane before reaching asymptotes at $\pm 60^{\circ}$. Due to the feedback on $i_{1}$, the complex poles start at a location farther in the left-half plane than for the conventional or WACC control law. Fig. 5 (in cyan) shows the root locus of the alternate control law when the inverter inductance $L_{2}=0.7 \hat{L}_{2}$ and $\hat{L}_{2}$ is the nominal inductance used in the control law. Because of the difference, the poles are slightly shifted, but because the poles start farther in the left-half plane, the effect is not sufficient to cause instability. The poles for both WACC and the algorithm proposed in this section are plotted for a wrong parameter estimation of $\hat{L}_{2}$ and a control gain $k_{P}=14.4128$ in Fig. 5 with a $*$. Note that the poles corresponding to the WACC algorithm turn to be unstable, but the new controller with the same gain remains stable.

\section{Example under unbalanced three-phase systems}

The complex description proposed in Section III is most effective for balanced three-phase systems. However, the method proposed in the paper could be extended to unbalanced systems by splitting the three-phase system into the positive and negative sequence components of the voltage and current 


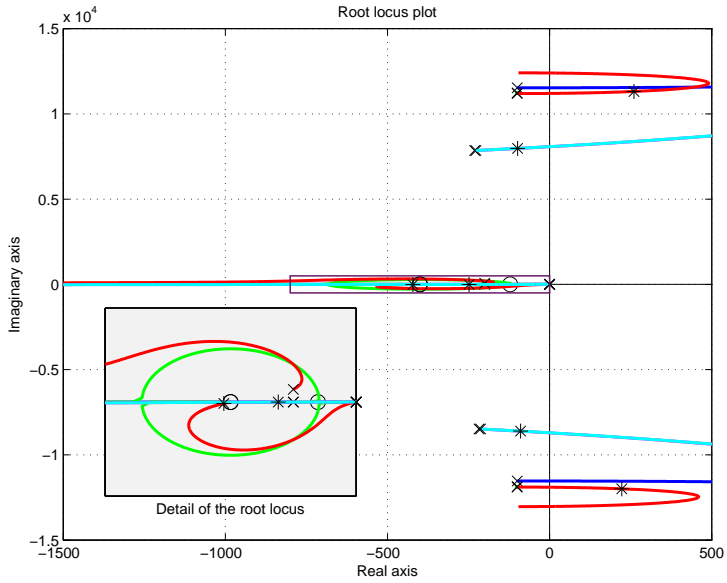

Fig. 8. Root locus comparing the conventional controller (in blue), the WACC (in green), the WACC with wrong parameters (in red), the alternative controller (in magenta) and the alternative controller with wrong parameters (in cyan). The poles for $k_{P}=14.4128$ corresponding to the WACC and alternative controllers with wrong parameters are plotted with *.
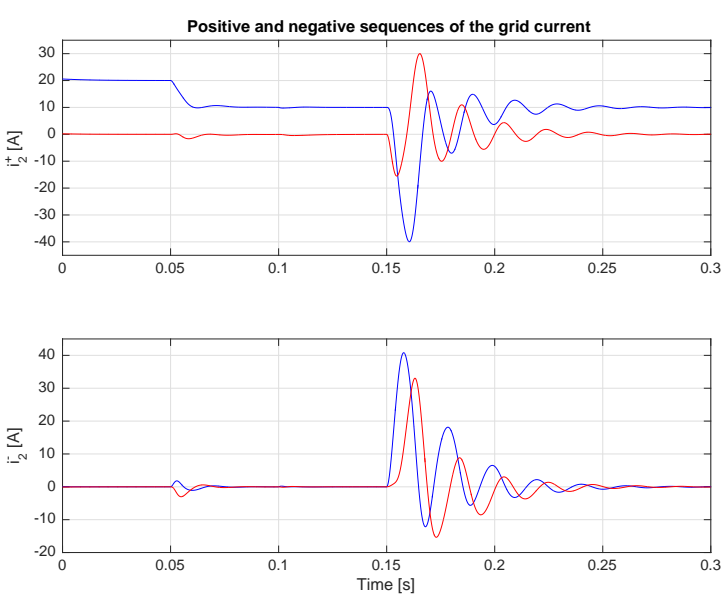

Fig. 9. Positive and negative sequences of the grid current. The real part correspond to the blue line, and the imaginary part to the red one.

vectors. Then, the controller extends to a double control loop that independently controls the positive and the negative sequences.

The simulation results for a $30 \mathrm{kV}$ inverter are shown in figures 9 and 10. A Dual Second Order Generalized Integrator-Frequency Locked Loop (DSOGI-FLL) [34], has been implemented in order to detect the magnitude of the symmetrical components. Then, two control loops with the form (84) are used for controlling the positive and negative sequences. For the simulation test the current references are set to $i_{2}^{+ \text {ref }}=20+j 0 \mathrm{~A}$ that changes to $i_{2}^{+ \text {ref }}=10+j 0 \mathrm{~A}$ at $t=0.05 \mathrm{~s}$, then the grid inductance is perturbed increasing up to $200 \%$ at $t=0.1 \mathrm{~s}$, and finally a $20 \%$ grid fault in phase C occurs at $t=0.15 \mathrm{~s}$. In all simulations $i_{2}^{- \text {ref }}=0+j 0 \mathrm{~A}$.

The simulation results show that the control design is robust against disturbances and is still valid for unbalanced threephase systems if the system is split into the symmetrical
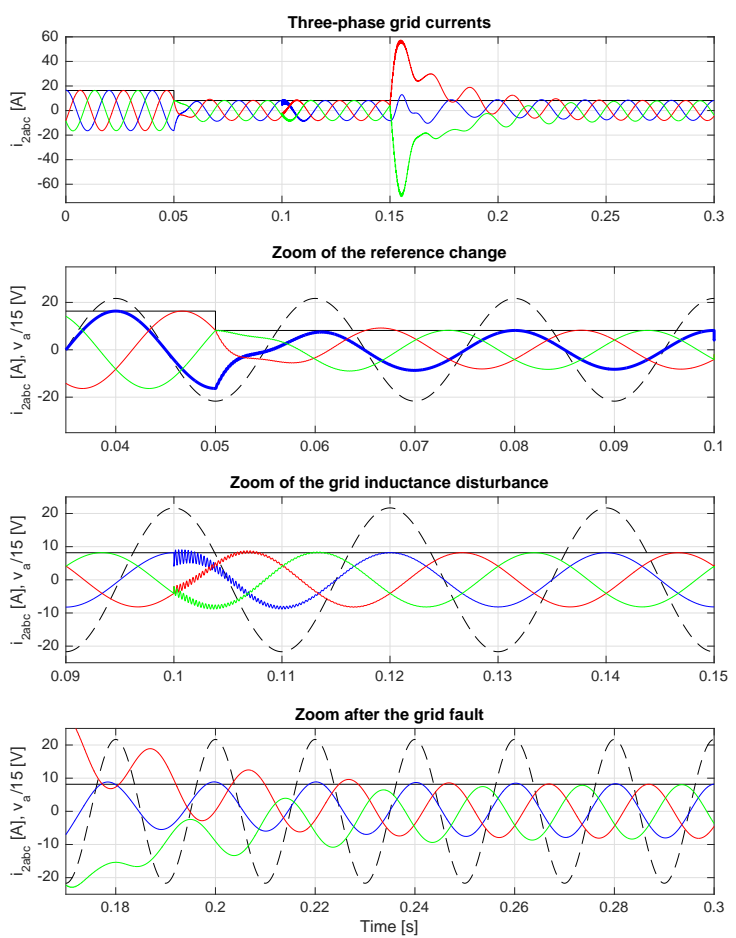

Fig. 10. Three-phase grid currents under the different scenarios. The dotted line corresponds to the grid voltage $v_{a} / 15$, the black line is the current reference amplitude and the three-phase currents are in blue (phase a), red (phase b) and green (phase c).

components. A further analysis on the whole unbalanced system is possible using complex transfer functions [10].

\section{CONCLUSiOnS}

The symmetric properties of balanced three-phase systems make it possible to describe their dynamics using an equivalent transfer function with complex coefficients. In this paper, we showed that the use of complex root locus rules could help in the design of feedback systems. In particular, the use of complex coefficients in the controller opens up a new range of possibilities to shape the branches of the closed loop root locus. The methodology was illustrated by using two examples: a DFIM and a three-phase LCL inverter.

Possible future works include the effect of unknown parameters and nonlinearities may perhaps be studied in the future using Robust Root Locus methods [35].

\section{REFERENCES}

[1] W. Evans, "Graphical analysis of control systems," AIEE Trans., vol. 67, no. 1, pp. 547-551, 1948.

[2] W. Evans, "Control systems synthesis by root locus method," AIEE Trans., vol. 69, no. 1, pp. 66-69, 1950.

[3] X. Zhang, J. Spencer, and J. Guerrero, "Small-signal modeling of digitally controlled grid-connected inverters with LCL filters," IEEE Trans. Ind. Electron., vol. 60, no. 9, pp. 3752-3765, 2013.

[4] F. Freijedo, A. Vidal, A. Yepes, J. Guerrero, O. López, J. Malvar, and J. Doval-Gandoy, "Tuning of synchronous-frame PI current controllers in grid-connected converters operating at a low sampling rate by MIMO root locus," IEEE Trans. Ind. Electron., vol. 62, no. 8, pp. 5006-5017, 2015. 
[5] B.-S. Chen and Y.-Y. Hsu, "A minimal harmonic controller for a STATCOM," IEEE Trans. Ind. Electron., vol. 55, no. 2, pp. 655-664, 2008.

[6] J. Muñoz, J. Espinoza, C. Baier, L. Morán, E. Espinosa, M. P.E, and D. Sárbaro, "Design of a discrete-time linear control strategy for a multicell UPQC," IEEE Trans. Ind. Electron., vol. 59, no. 10, pp. 37973807, 2012.

[7] A. Singh, A. Kaviani, and B. Mirafzal, "On dynamic models and stability analysis of three-phase phasor PWM-based CSI for stand-alone applications," IEEE Trans. Ind. Electron., vol. 62, no. 5, pp. 2698-2707, 2015.

[8] C. Choi and W. Lee, "Analysis and compensation of time delay effects in hardware-in-the-loop simulation for automotive PMSM drive system," IEEE Trans. Ind. Electron., vol. 59, no. 9, pp. 3403-3410, 2012.

[9] H. Mohammadpour and E. Santi, "Modeling and control of gatecontrolled series capacitor interfaced with a DFIG-based wind farm," IEEE Trans. Ind. Electron., vol. 62, no. 2, pp. 1022-1033, 2015.

[10] L. Harnefors, "Modeling of three-phase dynamic systems using complex transfer functions and transfer matrices," IEEE Trans. Ind. Electron., vol. 54, no. 4, pp. 2239-2248, 2007.

[11] F. B. del Blanco, M. Degner, and R. Lorenz, "Dynamic analysis of current regulators for AC motors using complex vectors," IEEE Trans. Ind. Appl., vol. 35, no. 5, pp. 1424-1423, 1999.

[12] M. Bodson and O. Kiselychnyk, "The complex Hurwitz test for the analysis of spontaneous self-excitation in induction generators," IEEE Trans. Autom. Control, vol. 58, no. 2, pp. 449-454, 2013.

[13] A. Dòria-Cerezo, M. Bodson, C. Batlle, and R. Ortega, "Study of the stability of a direct stator current controller for a doubly-fed induction machine using the complex Hurwitz test," IEEE Trans. Control Syst. Technol., vol. 21, no. 6, pp. 2323-2331, 2013.

[14] M. Illindala and G. Venkataramanan, "Frequency/sequence selective filters for power quality improvement in a microgrid," IEEE Trans. Smart Grid, vol. 3, no. 4, pp. 2039-2047, 2012.

[15] M. Barros and L. Lind, "On the splitting of a complex-coefficient polynomial," Proc. of the IEE, vol. 133, no. 2, pp. 95-98, 1986.

[16] M. Hromcik, M. Sebek, and J. Jezek, "Complex polynomials in communications: motivation, algorithms, software," in Proc. IEEE Int. Symposium on computer Aided Control Systems Design, 2002.

[17] N. Bose and Y. Shi, "A simple general proof of Kharitonov's generalized stability criterion," IEEE Trans. Circuits Syst., vol. 34, no. 8, pp. 12331237, 1987.

[18] D. Henrion, J. Jezek, and M. Sebek, "Efficient algorithms for discretetime symmetric polynomial equations with complex coefficients," in Proc. IFAC World Congress, 1990.

[19] E. Frank, "On the zeros polynomials with complex coefficients," Bulletin of the American Mathematical Society, vol. 5, no. 2, pp. 144-157, 1946.

[20] S. Agashe, "A new general Routh-like algorithm to determine the number of RHP roots of a real or complex polynomial," IEEE Trans. Autom. Control, vol. 30, no. 4, pp. 406-409, 1985.

[21] M. Benidir and B. Picinbono, "The extended Routh's table in the complex case," IEEE Trans. Autom. Control, vol. 36, no. 2, pp. 253-256, 1991.

[22] N. Bose, "Tests for Hurwitz and Schur properties of convex combination of complex polynomials," IEEE Trans. Circuits Syst., vol. 36, no. 9, pp. $1245-1247,1989$

[23] S. Chen and J. Tsai, "A new tabular form for determining root distribution of a complex polynomial with respect to the imaginary axis," IEEE Trans. Autom. Control, vol. 38, no. 10, pp. 1536-1541, 1993.

[24] Y. Bistritz, "Stability criterion for continuous-time system polynomial with uncertain complex coefficients," IEEE Trans. Circuits Syst., vol. 35, no. 4 , pp. $442-448,1988$

[25] N. Bose and Y. Shi, "Network realizability theory approach to stability of complex polynomials," IEEE Trans. Circuits Syst., vol. 34, no. 2, pp. 216-218, 1987.

[26] W. Karl and G. Verghese, "A sufficient condition for the stability of interval matrix polynomials," IEEE Trans. Autom. Control, vol. 38, no. 7, pp. 1139-1143, 1993.

[27] J. Kogan, "Robust Hurwitz $l^{p}$ stability of polynomials with complex coefficients," IEEE Trans. Autom. Control, vol. 38, no. 8, pp. 1304 1308, 1993.

[28] Y. Shi, K. Yen, and C. Chen, "Two necessary conditions for a complex polynomial to be strictly Hurwitz and their applications in robust stability analysis," IEEE Trans. Autom. Control, vol. 38, no. 1, pp. 125128,1993

[29] A. Dòria-Cerezo and M. Bodson, "Root locus rules for polynomials with complex coefficients," in Proc. 21st Mediterranean Conference on Control and Automation, 2013.
[30] M. Bodson, "Design of controllers in the complex domain," in Proc. 53th Conference on Decision and Control, 2014.

[31] K. Martin, "Complex signal processing is not complex," IEEE Trans. Circuits Syst. I, Reg. Papers, vol. 51, no. 9, pp. 1823-1836, 2004.

[32] C. Batlle, A. Dòria-Cerezo, and R. Ortega, "Power flow control of a doubly-fed induction machine coupled to a flywheel," European Journal of Control, vol. 11, no. 3, pp. 209-221, 2005.

[33] N. He, D. Xu, Y. Zhu, J. Zhang, G. Shen, Y. Zhang, J. Ma, and C. Liu, "Weighted average current control in a three-phase grid inverter with an LCL filter," IEEE Trans. Power Electron., vol. 28, no. 6, pp. 2785-2797, 2013.

[34] P. Rodríguez, A. Luna, I. Candela, R. Mujal, R. Teodorescu, and F. Blaabjerg, "Multiresonant frequency-locked loop for grid synchronization of power converters under distorted grid conditions," IEEE Trans. Ind. Electron., vol. 58, no. 1, pp. 127-138, 2011.

[35] Y. Tong and N. Sinha, "A computational technique for the robust root locus," IEEE Trans. Ind. Electron., vol. 41, no. 1, pp. 79-85, 1994.

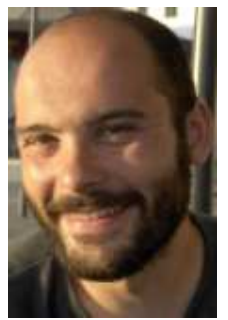

Arnau Dòria-Cerezo was born in Barcelona in 1974. He obtained his undergraduated in Electromechanical engineering and $\mathrm{PhD}$ degrees from Universitat Politècnica de Catalunya (UPC) in 2001 and 2006, respectively. He received his DEA in Industrial Automation from Laboratoire d'Automatisme Industrielle (LAI) at INSA-Lyon, France, in 2001. He is currently an Associate Professor at the Department of Electrical Engineering, and carries out his research with the research group on Advanced Control of Energy Systems at the Institute of Industrial and Control Engineering of the UPC. In 2003 and 2004 he was a Control Training Site-Research Fellow of Laboratoire des Signaux et Systèmes (L2S) at Supélec, France. In 2010, he was visitor at the Technische Universiteit Delft, Netherlands. Main research interests include control of electrical systems (related to its generation, conditioning, management and storage), modelling and advanced control of electrical machines and power converters, and modelling and control using passivity-based techniques.

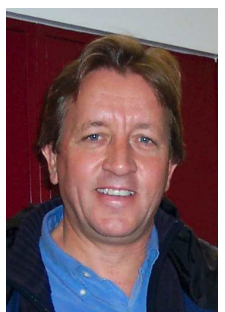

Marc Bodson (F'06) received a Ph.D. degree in Electrical Engineering and Computer Science from the University of California, Berkeley, in 1986. He obtained two M.S. degrees from the Massachusetts Institute of Technology in 1982. In 1980, he received the degree of Ingénieur Civil Mécanicien et Electricien from the Université Libre de Bruxelles, Belgium. Currently, he is a Professor of Electrical \& Computer Engineering at the University of Utah (Salt Lake City). He was Chair of the department between 2003 and 2009, and he was the Editor-inChief of IEEE Trans. on Control Systems Technology from 2000 to 2003. He was elected Fellow of the IEEE in 2006, and Associate Fellow of the AIAA in 2013. His research interests are in adaptive control, with applications to electromechanical systems and aerospace. He was a Belgian American Educational Foundation Fellow in 1980 and a Lady Davis Fellow at the Technion, Haifa, Israel, in 1990. 